Articulation emploi-famille et temps de travail :

De quelles mesures disposent les travailleurs canadiens et à quoi aspirent-ils?

\author{
Diane-Gabrielle Tremblay \\ Elmustapha Najem \\ Renaud Paquet
}

Note de recherche de la Chaire de recherche du Canada sur les enjeux socio-organisationnels de l'économie du savoir

No 2006-02

Télé-université

Université du Québec à Montréal

Janvier 2006 
Distribution :

Chaire de recherche sur les enjeux socio-organisationnels de l'économie du savoir

Télé-université

Université du Québec à Montréal

100 rue Sherbrooke Ouest, Montréal, Québec, Canada H2X 3P2

Téléphone : 514-843-2015

Fax : 514-843-2160

Courriel : dgtrembl@teluq.uquebec.ca

\section{Note biographique}

Diane-Gabrielle Tremblay Titulaire de la chaire de recherche du Canada sur les enjeux socio-organisationnels de l'économie du savoir et cotitulaire de la Chaire Bell-Téluq-Enap en technologies et organisation du travail. Elle est professeure à la Télé-Université de l'Université du Québec à Montréal, et elle a été professeure invitée à l'université de Paris I Sorbonne, à l'université de Lille I, à la European School of Management et à l'université de Louvain-la-Neuve, en Belgique. Elle est membre du comité sur la sociologie du travail de l'Association internationale de sociologie, du conseil exécutif de la Society for the Advancement of Socio-Economics et codirectrice du comité sur les temps sociaux de l'Association internationale des sociologues de langue française. Elle est également présidente de l'Association d'économie politique et rédactrice en chef de la revue électronique Interventions économiques. (www.teluq.uquebec.ca/interventionseconomiques) Elle a en outre écrit plusieurs ouvrages et rédigé divers articles portant sur la nature du travail et sur les types d'emplois, la formation en entreprise, les innovations dans l'organisation du travail et du lieu de travail (télétravail), de même que sur l'articulation de la vie professionnelle et familiale. Voir :

www.teluq.uquebec.ca/chaireecosavoir www.teluq.uquebec.ca/chairebell www.teluq.uquebec.ca/interventionseconomiques www.teluq.uquebec.ca/chaireecosavoir/cvdgt;

www.teluq.uquebec.ca/ dgtrembl

e-mail : dgtrembl@teluq.uquebec.ca

Note : Ce texte a été proposé à une revue pour publication en 2006. 


\title{
Articulation emploi-famille et temps de travail : De quelles mesures disposent les travailleurs canadiens et à quoi aspirent-ils?
}

\author{
Diane-Gabrielle Tremblay \\ Elmustapha Najem \\ Renaud Paquet ${ }^{1}$
}

Plusieurs recherches des dernières années montrent que bon nombre de parents qui occupent un emploi parviennent difficilement à concilier leurs obligations professionnelles et familiales et se sentent stressés de ce fait (Nelson et Quick, 1985; Galinsky et al., 2001; Tremblay, 2004, 2002, Tremblay et Amherdt, 2000; Tremblay et de Sève, 2002; Paquet et Najem, 2005). En effet, l'organisation de la vie et du temps hors du travail est grandement affectée par les transformations qu’a connu le marché du travail durant les trente dernières années. Ces changements qui se manifestent notamment par une intensification du travail, par la diversification des formes d'emplois (temporaire, autonome, sur appel, occasionnel) et d’horaires (temps partiel, horaires brisés, etc.) viennent accroître d’autant les difficultés qu’ont les parents à articuler emploi et famille.

Il existe toute une panoplie de recherches, développées principalement en Europe, dont l'axe central porte sur la dimension temporelle du rapport entre la vie de travail et la vie hors travail et les difficultés de conciliation qui en résultent. Certaines de ces études montrent que les mesures de conciliation emploi-famille les plus recherchées par les parents ayant de jeunes enfants ont trait à la flexibilité des horaires (Lero et al., 1993, Tremblay, 2004). En s’inscrivant dans la lignée de ces travaux, nous nous intéressons, dans cet article, aux horaires de travail et à l'incidence des mesures de conciliation axées sur l'aménagement du temps de travail ainsi que d'autres types de mesures de conciliation chez les travailleurs et les travailleuses canadiens.

Alors que les enquêtes menées sur la conciliation emploi-famille portent généralement sur des échantillons réduits, souvent quelques centaines de personnes (Descarries et Corbeil, 1995, 1996; Descarries et al., 1995a,b,c; Guérin et al., 1997; Tremblay et De Sève, 2002), ce qui présente

\footnotetext{
${ }^{1}$ Diane-Gabrielle est professeure à la Télé-université de l'UQÀM et titulaire de la Chaire de recherche du Canada sur les enjeux socio-organisationnels de l'économie du savoir (www.teluq.uquebec.ca/chaireecosavoir). Elmustapha Najem et Renaud Paquet sont professeurs au Département de relations industrielles de l’Université du Québec en Outaouais et chercheurs au Centre d'études et de recherche sur l'emploi, le syndicalisme et le travail. Les auteurs remercient la division de l'Enquête sur le milieu de travail et les employés (EMTE) de Statistique Canada qui leur a fourni l'accès aux données originales de l’EMTE sur laquelle repose une partie de cet article.
} 
l'avantage de traiter uniquement de la situation des parents, mais par contre d'être possiblement moins représentatif de l'ensemble de la population canadienne, nous avons voulu exploiter les données de l'Enquête sur le milieu de travail et les employés (EMTE) pour évaluer la situation globale à cet égard au Canada à l'aide de données statistiques représentatives, qui n’ont pas été exploitées à ce jour sous cet angle. ${ }^{2}$ Le reste du texte se divise en trois parties. La première porte sur une recension des écrits; la deuxième traite des données utilisées et la troisième présente les résultats de la recherche. Nous discutons de la portée et des limites de nos principales constatations dans une conclusion générale.

\section{Recension des écrits}

Il est difficile de rendre compte succinctement de tout ce qui a été écrit, au cours des dernières années, sur cette problématique de l'articulation ou du « conflit » ${ }^{3}$ emploi-famille, ainsi que des stresseurs associés à cette situation. Nous exposons ici les principaux écrits pertinents en regard de l'objet d'étude identifié plus haut. Ainsi, certaines recherches estiment que les caractéristiques de l'emploi sont déterminantes dans le conflit emploi-famille (Guérin et al., 1997); alors que d'autres mettent plutôt l'accent sur l'importance de la catégorie socio-professionnelle comme facteur de différenciation de la participation des hommes et des femmes aux responsabilités parentales comme aux responsabilités professionnelles (Nelson et Quick, 1985). Ces deux éléments étant nécessairement des vases communicants, une forte implication dans l'un se fait souvent au détriment de l'autre. Dans certains couples, il y a bien sûr équilibre et participation équivalente, mais ce n'est généralement pas le cas, même si la participation des hommes aux activités familiales s'est accrue au Québec et au Canada (Pronovost, 2005).

Comme nous l'avons indiqué précédemment, plusieurs recherches témoignent des sentiments de manque de temps exprimés par les parents de jeunes enfants et démontrent qu’une forte proportion des parents qui occupent un emploi se sentent stressés parce qu'ils parviennent

\footnotetext{
${ }^{2}$ Ceci constitue la première partie d'une recherche comparative France-Canada visant à déterminer les mesures de conciliation existant dans les deux pays et leur impact potentiel sur l'activité et les heures de travail des femmes. Cette recherche est financée par la DARES (Direction de l'analyse, de la recherche et des études statistiques) du ministère du travail de la France, que nous remercions.

${ }^{3}$ Ce terme est plus souvent employé par les psychologues, qui renvoient au «conflit » de rôles; ce n’est pas nécessairement la meilleure façon d'aborder le phénomène de l'articulation emploi-famille, comme nous l'avons observé dans la partie qualitative (entrevues) de la recherche. En effet, les parents se reconnaissent généralement peu dans cette expression. Nous l'employons néanmoins ici lorsque les auteurs l’ont eux-même utilisée.
} 
difficilement à concilier leurs obligations professionnelles et familiales. D’après Carlson et al. (2000), les études menées jusqu’à maintenant ont permis de dégager trois formes de conflit: les conflits de temps, de tension et de comportement. Le conflit de temps découle de la surcharge due aux multiples rôles et de la difficulté à coordonner les exigences concurrentielles de chacun d'eux, et c'est surtout à ce premier conflit que nous nous intéressons ici. Précisons toutefois que le conflit de tension provient d'une tension vécue dans un rôle, laquelle s'insère dans sa participation à un autre rôle et vient interférer avec ce dernier. Le conflit de comportement se produit lorsque le comportement spécifique à un rôle est incompatible avec le comportement attendu dans un autre rôle et que les ajustements nécessaires ne sont pas réalisés par la personne. Ces deux derniers éléments intéressant surtout les psychologues, nous nous intéressons davantage à la conciliation emploi-famille du point de vue des aménagements temporels possibles.

Les facteurs influant sur l'ampleur du conflit emploi-famille peuvent être regroupés en trois grandes catégories, soit le travail, les employés, et la famille. En ce qui concerne le facteur travail, on tient compte de l'environnement de travail et des caractéristiques de l'emploi, dont l'existence de pratiques de conciliation «emploi-famille ». Parmi les autres caractéristiques de l'emploi on retrouve le type d'emploi, la nature des tâches et des rôles, le nombre d'heures travaillées par semaine et l’horaire de travail; ces deux derniers éléments s’étant révélés déterminants dans plusieurs études (Guérin et al., 1997; Tremblay, 2004, 2004b).

En se basant sur une recension des écrits, Nelson et Quick (1985) présentent un modèle des agents stresseurs chez les femmes professionnelles en emploi. On y retrouve un bon nombre de variables liées à l’organisation du travail, mais aussi des éléments associés au poste spécifique de la personne. Une autre étude réalisée en 2001 auprès d'un échantillon de 1003 travailleurs américains âgés de 18 ans et plus (Galinsky et al., 2001) s’est penchée sur le facteur de la surcharge de travail en évaluant les différents aspects de l'emploi qui procurent cette impression chez les travailleurs : le nombre d'heures travaillées par semaine et leurs préférences, le nombre de jours travaillés par semaine et leurs préférences et, finalement, les employés ayant la conviction de ne pas pouvoir adapter leur horaire de travail (en heures et en jours) en fonction de leurs préférences. En somme, c’est en grande partie l'écart entre les attentes et ce qui est offert 
qui est à la source du problème. Ce dernier aspect nous intéresse plus particulièrement et nous y reviendrons lors de la présentation des résultats à la troisième section de cet article.

Au Québec, l'étude de Guérin et al. (1997) auprès de 1345 employés syndiqués de la CSN ayant au moins un enfant à charge (75,6 \% de femmes et 24,3\% d'hommes) portait sur l'effet de la nature du travail, des conflits de rôles, de l'ambiguïté du travail, de la surcharge du travail, de l'horaire et des déplacements pour se rendre au travail sur le niveau de stress des employés; la surcharge et les horaires de travail sont ressortis comme variables déterminantes.

D’autres auteurs se sont intéressés aux caractéristiques de l'emploi. L'organisation du temps de travail (horaires longs, rotation, journées ou heures de travail changeantes) constitue un agent stresseur pour les employés de nombreux secteurs d'activité puisqu'elle influence leur vie au travail tout en perturbant leur vie extraprofessionnelle et en fragilisant souvent leur état de santé (Families and work institute, 1998, Galinsky et al., 2001). Nous avons donc voulu analyser ces dimensions des heures de travail, de la rotation et des changement de jours et d'heures de travail en traitant les données de l’EMTE sur ce sujet.

En ce qui concerne la famille, il est possible d'identifier plusieurs des facteurs stressants dans la famille. Guérin et al. (1997) ont identifié certains facteurs reliés aux enfants comme étant déterminants dans le conflit (nombre, âge moyen, présence d'un enfant handicapé, charge totale due aux enfants). Cette étude montre que le conflit emploi-famille augmente avec le nombre d'enfants dont on est responsable et c'est pourquoi notre analyse des données de l'EMTE sera différenciée selon le nombre d'enfants.

Nous avons aussi recensé des écrits portant sur l'influence de la présence de mesures d'aide à l'employé et à sa famille. Parmi les pratiques d'aide à la conciliation, Guérin et al. (1994) ont identifié les garderies, le temps partiel volontaire et le travail à domicile, les compléments de salaire et de congés à la naissance. Nous nous intéressons donc aux services de garde offerts par les organisations, à la semaine de travail réduite ou comprimée, ainsi qu'au travail à domicile. En effet, les données que nous présentons dans cet article permettront une dizaine d'années plus tard 
de voir comment ces pratiques se sont étendues et qui en bénéficient. En fait, comme nous l'avons observé (Paquet et Najem, 2004; Tremblay, 2004), il existe souvent un écart important entre la présence d'une pratique dans une entreprise et la possibilité offerte aux salariés de s’en prévaloir, de là l’importance d’en analyser l’incidence.

\section{Méthodologie et source des données}

Dans le but d'offrir une réponse éclairée aux questions posées par le présent article, nous avons eu recours aux données de l'Enquête sur le milieu de travail et les employés (EMTE) de Statistique Canada, qui offrent aux chercheurs des données empiriques représentatives du marché du travail canadien. Pour les fins de la présente recherche, nous utilisons, sur une base fusionnée, les données recueillies auprès des employeurs et les réponses fournies par les employés à l'enquête. En effet, l'EMTE est constituée de deux banques de données, celle des employeurs et celle des employés contenant ensemble plusieurs centaines d'indicateurs. L’EMTE vise un échantillon représentatif du marché du travail canadien, à l'exception des fonctions publiques fédérale, provinciales et municipales qui en sont exclues. Les données qu'elle comprend ont été recueillies auprès de plus 6000 entreprises et quelques 23000 salariés de ces mêmes entreprises (Statistique Canada, 2004). Les banques de données sont construites de façon à pouvoir être fusionnées afin d'obtenir l'ensemble des informations disponibles sur un employé et l'entreprise qui l'emploie. Les résultats présentés dans la prochaine section résultent justement de la fusion de ces deux banques.

Pour les fins de cette recherche, nous avons utilisé les résultats pondérés qui permettent une généralisation des données au marché du travail canadien, à l'exception comme nous en avons déjà fait mention des fonctions publiques. Les données utilisées sont celles des enquêtes de 1999 et de 2002. Sur une base longitudinale, il s’agit là d'une période relativement courte (4 ans), mais qui permet quand même de bien saisir les tendances du marché du travail, d'autant plus que ces quatre années ont été marquées par un important débat sociétal sur la question du temps de travail et de la conciliation travail-famille. Si les pratiques en entreprise ont suivi la tendance sociale des idées et du débat, on devrait constater des changements au cours de la période analysée. 
Les données de 1999 ont été choisies car il s'agissait de la première année de l'EMTE. À l'été 2005, au moment de l’analyse, les données de 2002 étaient les dernières données disponibles, les données des enquêtes de 2003 et 2004 n’étant pas encore accessibles aux chercheurs. Mentionnons aussi que le logiciel SAS a été utilisé pour le pairage des deux banques de données (employeurs et employés), pour le recodage des informations et la construction des variables composées ainsi que pour les analyses statistiques univariées et bivariées qui sont ici présentées. Les résultats obtenus ont été soigneusement revus afin de nous assurer d’une part, que les contraintes imposées par Statistique Canada étaient respectées et d’autre part, que des erreurs ne s’étaient pas glissées lors de la programmation ou de la compilation.

Notons que lors de la présentation des résultats issus des croisements de variables, nous ne présentons pas les coefficients et les degrés de signification compte tenu que le nombre de répondants, une fois l'échantillon pondéré, dépasse les 11 millions, rendant ainsi tous les écarts comparatifs significatifs sur le plan statistique.

\section{Résultats}

Tenant compte de notre recension des écrits, nous présenterons dans cette partie les analyses qui permettent de vérifier si les horaires de travail varient selon le sexe ou le nombre d'enfants, et si les personnes ayant des charges familiales ont des horaires plus longs ou plus courts que les autres. Nous nous pencherons ensuite sur les aspirations des individus relatives au temps de travail (souhaitent-ils un allongement ou une réduction) et en particulier aux motifs qui peuvent expliquer l’intérêt pour une réduction du temps de travail, notamment les obligations familiales. Enfin, nous examinerons l'incidence des mesures d'aménagement du temps de travail, dont la semaine réduite ou comprimée, mesure connue comme pouvant favoriser une certaine conciliation. Nous regarderons à ce moment comment la situation se présente selon le sexe et selon le nombre d'enfants. Dans cette dernière section sur les mesures facilitant la conciliation, nous présenterons aussi les données sur les mesures offertes en matière de services de garde des enfants ou de soins aux aînés.

\subsection{Les heures de travail selon le sexe et selon le nombre d'enfants}


Le tableau 1 expose les heures habituellement travaillées selon le sexe en 1999 et 2002. On y observe que les femmes travaillent habituellement moins d'heures par semaine que les hommes, soit 33,88 heures en 2002 pour les femmes contre 39,51 heures pour les hommes durant la même année, ce qui pourrait nous amener à penser que plusieurs d'entre elles réduisent leurs heures de travail en vue de concilier emploi et famille. Nous verrons plus loin qu'elles sont d'ailleurs plus nombreuses que les hommes à souhaiter réduire leurs heures de travail, et que c'est plus souvent pour des raisons familiales. Au cours de la même période, on note que les heures supplémentaires non rémunérées ou rémunérées se sont réduites. Par contre, le nombre de jours de travail par semaine s'est légèrement accru, se rapprochant fortement de 5 jours (4,93 en moyenne pour les hommes et à 4,6 pour les femmes en 2002).

Le fait de travailler à la maison est parfois perçu comme une façon de concilier ses responsabilités familiales et professionnelles (Tremblay, 2002), quoique cela peut aussi contribuer à rendre plus floues les frontières entre le temps de travail et le temps personnel ou familial, ce qui peut aussi être considéré comme une intrusion négative du travail dans la vie privée. (Baines et Gelder, 2003). Entre 1999 et 2002, on observe, sur ce plan, une hausse des heures de travail à la maison autant pour les hommes que pour les femmes, les deux groupes accomplissant respectivement, en moyenne, 6,14 et 6,13 heures de travail à la maison. Ceci est important, puisque cela correspond à près d'une journée par semaine.

\section{Tableau 1}

Les heures de travail selon le sexe, 1999-2002

\begin{tabular}{|l|c|c|c|c|}
\hline & $\begin{array}{c}\text { Femmes } \\
\mathbf{1 9 9 9}\end{array}$ & $\begin{array}{c}\text { Hommes } \\
\mathbf{1 9 9 9}\end{array}$ & $\begin{array}{c}\text { Femmes } \\
\mathbf{2 0 0 2}\end{array}$ & $\begin{array}{c}\text { Hommes } \\
\mathbf{2 0 0 2}\end{array}$ \\
\hline $\begin{array}{l}\text { Heures habituellement } \\
\text { travaillées }\end{array}$ & 33,97 & 39,51 & 33,88 & 39,51 \\
\hline \hline Heures supp. non rémunérées & 1,71 & 2,42 & 1,26 & 1,65 \\
\hline \hline Heures supp. rémunérées & 0,51 & 1,63 & 0,13 & 1,17 \\
\hline Nb jours travail dans semaine & 4,11 & 4,53 & 4,60 & 4,93 \\
\hline
\end{tabular}




\section{Hres hebdo. de travail à la}

maison

\section{5,34}

5,80

6,14

6,13

Si plusieurs études rapportent un effet non négligeable du nombre d'enfant sur les difficultés de conciliation, les données des tableaux $2 \mathrm{a}$ et $2 \mathrm{~b}$, permettent de constater que cet effet est à tout le moins ambigu. En effet, si l'augmentation du nombre d'enfants semble se traduire par une augmentation des heures supplémentaires non rémunérées jusqu’à deux enfants, il y a ensuite une légère réduction pour ceux ayant trois enfants. Pour ce qui est des heures supplémentaires rémunérées, on constate qu'elles ont tendance à croître avec le nombre d'enfants et qu'elles sont , toutefois, moins nombreuses que les heures non rémunérées et ce autant en 1999 qu’en 2002.

L'effet sur le travail à domicile est encore plus nuancé. Si la présence d'un enfant se traduit par une augmentation par rapport à l'absence d'enfants, les chiffres reculent pour les individus ayant 2 enfants et encore davantage pour ceux ayant 3 enfants. Il semble donc que la présence d'enfants plus nombreux tende à réduire le nombre d'heures de travail à domicile.

Tableau 2a

Les heures de travail selon le nombre d'enfants, 1999

\begin{tabular}{|c|c|c|c|c|}
\hline 1999 & Aucun & 1 enfant & 2 enfants & 3 et plus \\
\hline $\begin{array}{l}\text { Heures habituellement } \\
\text { travaillées }\end{array}$ & 35,88 & 38,01 & 37,09 & 37,35 \\
\hline Heures supp. non rémunérées & 1,86 & 2,06 & 2,45 & 2,14 \\
\hline Heures supp. rémunérées & 0,91 & 1,09 & 1,15 & 1,55 \\
\hline Nb jours travail dans semaine & 4,26 & 4,43 & 4,22 & 4,21 \\
\hline $\begin{array}{l}\text { Hres hebdo. de travail à la } \\
\text { maison }\end{array}$ & 5,31 & 6,75 & 5,40 & 5,38 \\
\hline
\end{tabular}


Tableau 2b

Les heures de travail selon le nombre d'enfants, 2002

\begin{tabular}{|c|c|c|c|c|}
\hline 2002 & Aucun & 1 enfant & 2 enfants & 3 et plus \\
\hline $\begin{array}{l}\text { Heures habituellement } \\
\text { travaillées }\end{array}$ & 36,17 & 37,36 & 37,25 & 36,81 \\
\hline Heures supp. non rémunérées & 1,32 & 1,51 & 1,74 & 1,40 \\
\hline Heures supp. rémunérées & 0,51 & 0,66 & 0,91 & 0,75 \\
\hline Nb jours travail dans semaine & 4,74 & 4,81 & 4,80 & 4,73 \\
\hline $\begin{array}{l}\text { Hres hebdo. de travail à la } \\
\text { maison }\end{array}$ & 6,45 & 6,67 & 5,81 & 4,92 \\
\hline
\end{tabular}

Par ailleurs, certains arrangements du temps de travail peuvent être source de difficultés supplémentaires, comme l’ont indiqué des travaux évoqués dans la recension des écrits plus haut. Les horaires rotatifs, les heures ou jours de travail qui changent peuvent aussi présenter des défis pour les personnes qui cherchent à concilier leurs obligations personnelles et familiales.

Les données de l'EMTE présentées au tableau 3, indiquent qu'entre 1999 et 2002, il y a eu une augmentation des quarts de travail rotatifs autant pour les femmes que pour les hommes et que cette hausse est davantage accentuée chez les premières. Par ailleurs, les femmes travaillent davantage les mêmes jours dans la semaine et les mêmes heures dans la semaine que ce n’est le cas chez les hommes en 2002, alors que c’était l'inverse en 1999. Les données sont relativement peu diversifiées selon le nombre d'enfants, si ce n’est le fait qu'en 2002, les quarts de travail rotatifs sont moins fréquents au fur et à mesure que le nombre d'enfants augmente, passant de 53 \% en présence d’un enfant, à 47 \% lorsqu’il y a deux enfants, puis à 40 \% lorsqu’ils sont trois. 
Tableau 3

Les horaires de travail diversifiés, selon le sexe, 1999-2002

\begin{tabular}{|l|c|c|c|c|}
\hline & Femmes 1999 & Hommes 1999 & Femmes 2002 & Hommes 2002 \\
\hline Horaires rotatifs & 34,11 & 46,40 & 48,09 & 48,73 \\
\hline $\begin{array}{l}\text { Mêmes heures } \\
\text { chaque jour }\end{array}$ & 56,00 & 60,99 & 76,82 & 64,98 \\
\hline $\begin{array}{l}\text { Mêmes jours } \\
\text { dans la semaine }\end{array}$ & 54,49 & 65,43 & 68,72 & 66,95 \\
\hline
\end{tabular}

Nous pouvons conclure qu'un pourcentage important de la main-d'œuvre canadienne vit des horaires de travail variables et des horaires rotatifs, ce qui a été identifié comme source de difficultés de conciliation dans d'autres recherches (Guérin et al., 1997; Tremblay et De Sève, 2002). Les heures supplémentaires ne sont généralement pas très importantes, et les individus peuvent apparemment travailler un certain nombre d'heures à domicile, ce qui peut favoriser la conciliation, mais peut aussi être source d'empiètement sur la vie privée (Taskin et Vendramin, 2004)

\subsection{Les aspirations en matière de temps de travail}

Les aspirations en matière de temps de travail ont été l'objet de nombreux débats au Canada comme ailleurs. Une enquête menée dans les années 90 par Développement des ressources humaines Canada indiquait que seulement 6\% de la population canadienne souhaiterait réduire sa durée de travail. ${ }^{4}$ Par ailleurs, une enquête menée sur ce sujet par la CEQ dans les années 90 fait ressortir le fait qu'avec une compensation salariale partielle, 66\% des membres de la Centrale de l'Enseignement du Québec étaient favorables à une réduction de leur temps de travail. Enfin, 48,8\% des personnes interrogées dans le cadre d'une enquête menée par la F.T.Q. acceptaient de

\footnotetext{
${ }^{4}$ Développement des ressources humaines Canada, « Les Canadiens veulent travailler davantage, et non moins », (199???????????)
} 
réduire leur temps de travail sans compensation ou avec une compensation partielle de leur salaire. Comme les deux dernières enquêtes ont été menées sur de petits échantillons non représentatifs de la population en général, nous avons voulu les comparer aux données de l'EMTE ,d'autant plus que ces dernières présentent également des indications sur les motifs qui fondent le désir de la réduction du temps de travail, notamment pour obligations familiales.

Ainsi, le tableau 4 montre que la majorité des gens sont satisfaits de leurs heures de travail, puisque autant en 1999 qu'en 2002, il n'y a qu'environ un homme et une femme sur cinq qui souhaiteraient des heures de travail supplémentaires, alors qu'environ $10 \%$ de chacune des deux catégories désiraient les réduire en 1999, et seulement 7,6 \% des hommes et 7,95 \% des femmes souhaitaient les réduire en 2002. En ce qui concerne les motifs invoqués pour souhaiter la réduction du temps de travail, les obligations familiales viennent au premier rang pour les femmes (47 \% en 1999 et $59 \%$ en 2002 ), alors que les hommes qui invoquent cette raison sont relativement moins nombreux que les femmes. Toutefois, il y a lieu de noter la hausse du pourcentage d'hommes qui souhaitent réduire leurs heures de travail en raison de leurs obligations familiales, ce pourcentage étant passé de $36 \%$ à $42 \%$ au cours de la même période. Enfin, il faut noter que d'autres travaux avaient permis de constater que la variable salariale et la réduction potentielle du revenu font que nombre d'individus ne peuvent se permettre une réduction du temps de travail, même s'ils pourraient le souhaiter.

\section{Tableau 4}

Les aspirations en matière de temps de travail selon le sexe, 1999-2002

\begin{tabular}{|l|c|c|c|c|} 
& $\begin{array}{c}\text { Femmes } \\
\mathbf{1 9 9 9}\end{array}$ & $\begin{array}{c}\text { Hommes } \\
\mathbf{1 9 9 9}\end{array}$ & $\begin{array}{c}\text { Femmes } \\
\mathbf{2 0 0 2}\end{array}$ & $\begin{array}{c}\text { Hommes } \\
\mathbf{2 0 0 2}\end{array}$ \\
\hline Veut heures additionnelles & 19,55 & 18,62 & 18,33 & 21,89 \\
\hline Veut réduire ses heures & 9,13 & 10,37 & 7,95 & 7,60 \\
\hline Motifs : Obligations familiales & 47,75 & 36,03 & 58,92 & 41,99 \\
\hline \hline Stress lié au travail & 17,90 & 17,96 & 33,45 & 28,74 \\
\hline
\end{tabular}




\begin{tabular}{|l|c|c|c|c|}
\hline Raisons de santé & 5,82 & 4,58 & 17,60 & 12,99 \\
\hline \hline Plus de loisirs & 47,24 & 61,29 & 56,52 & 62,82 \\
\hline \hline Autres & 11,16 & 14,30 & 7,19 & 10,65 \\
\hline
\end{tabular}

Par ailleurs, les données des tableaux $5 \mathrm{a}$ et $5 \mathrm{~b}$ font ressortir le fait que la présence d'enfants a une incidence sur le désir de réduire son temps de travail, mais celle-ci est aussi ambiguë. Les personnes avec 3 enfants et plus sont moins nombreuses à vouloir augmenter leur temps de travail, mais elles sont aussi moins nombreuses à vouloir le réduire. Par contre, lorsqu'elles souhaitent réduire leur temps de travail, les personnes ayant des enfants invoquent le plus souvent les obligations familiales pour justifier leur intérêt à réduire leur temps de travail.

Tableau 5a

Les aspirations en matière de temps de travail, selon le nombre d'enfants, 1999

\begin{tabular}{|c|c|c|c|c|}
\hline 1999 & Aucun & 1 enfant & 2 enfants & 3 et plus \\
\hline Veut heures additionnelles & 20,87 & 17.09 & 18.04 & 15.01 \\
\hline Veut réduire ses heures & 8,42 & 10.59 & 12.02 & 10.23 \\
\hline Motifs :Obligations familiales & 22,77 & 46.37 & 63.06 & 63.50 \\
\hline Stress lié au travail & 18,77 & 20.48 & 13.47 & 21.85 \\
\hline Raisons de santé & 5,66 & 7.55 & 3.36 & 3.78 \\
\hline Plus de loisirs & 62,46 & 50.93 & 44.70 & 49.98 \\
\hline Autres & 18,17 & 9.42 & 8.63 & 4.82 \\
\hline
\end{tabular}

Tableau $5 b$

Les aspirations en matière de temps de travail, selon le nombre d'enfants 2002

\begin{tabular}{|l|c|c|c|c|}
\hline $\mathbf{2 0 0 2}$ & Aucun & 1 enfant & 2 enfants & 3 et plus \\
\hline \hline Veut heures additionnelles & 22,03 & 19,63 & 17,67 & 15,50 \\
\hline
\end{tabular}




\begin{tabular}{|l|c|c|c|c|}
\hline Veut réduire ses heures & 6,57 & 9,07 & 9,47 & 8,22 \\
\hline \hline Motifs :Obligations familiales & 29,33 & 65,96 & 72,14 & 58,56 \\
\hline Stress lié au travail & 34,10 & 34,85 & 21,79 & 36,88 \\
\hline Raisons de santé & 21,86 & 8,43 & 9,51 & 16,11 \\
\hline \hline Plus de loisirs & 66,19 & 53,03 & 58,75 & 43,98 \\
\hline Autres & 12.16 & 6,06 & 5,09 & 9,99 \\
\hline
\end{tabular}

\subsection{Les mesures de conciliation}

Dans cette section, nous traitons d'abord des mesures de conciliation axées sur l'aménagement du temps de travail, qui ont souvent été identifiées comme le principal facteur facilitateur recherché par les parents (Lero et al., 1993; Tremblay et Amherdt, 2000). Puis nous nous intéressons aux services de garde et aux soins aux aînés, les premiers ayant été identifiés comme demande principale des parents canadiens d'enfants de moins de 3 ans, et les seconds apparaissant de plus en plus importants puisqu'un nombre croissant de travailleurs doivent aujourd'hui s'occuper d'un parent vieillissant (Lero et al., 1993; Tremblay, 2004)

\subsubsection{Les mesures de conciliation axées sur l'aménagement du temps de travail}

Nous avons mentionné plus haut que le fait de travailler à la maison est parfois perçu comme une façon de concilier ses responsabilités familiales et professionnelles (Tremblay, 2002), mais que cela peut aussi être perçu comme une intrusion négative du travail dans la vie privée (Baines et Gelder, 2003; Taskin et Vendramin, 2004). Nous avons observé, à partir des données du tableau 2, qu'entre 1999 et 2002, une hausse des heures de travail à la maison autant pour les hommes que pour les femmes, les deux groupes accomplissant respectivement, en moyenne, 6,14 et 6,13 heures de travail à la maison. Ceci est important, puisque cela correspond à près d'une journée par semaine.

Pour leur part, les données du tableau 6 indiquent que tant pour les hommes que pour les femmes, le travail à domicile s'explique surtout par les exigences du travail, qu'évoquent environ les deux tiers des personnes qui font du travail à la maison. Il ne semble donc pas que le travail à domicile 
soit utilisé prioritairement comme une manière de concilier les exigences professionnelles et familiales.

Il est intéressant de noter par ailleurs que le motif de la conciliation a reculé chez les femmes entre 1999 et 2002 (de 6,08 à 4,71 \% des femmes pratiquant le travail à domicile)) alors qu'il augmentait chez les hommes (2,28 à 6,75 \% des hommes pratiquant le travail à domicile). Comme les travaux sur le télétravail indiquent que les hommes font surtout du travail à domicile occasionnel pour réaliser le travail qu’ils n’arrivent pas à accomplir au bureau, en tant que professionnel ou gestionnaire généralement (Taskin et Vendramin, 2004), on peut penser que c'est pour être présent à la maison, même s’ils ne sont pas disponibles pour la famille à ce moment, qu'ils choisissent de faire plus de travail à la maison. On peut aussi souligner que les hommes sont de plus en plus sensibles à la question de la conciliation (Politiques sociales, 2003; Tremblay, 2003), de sorte qu'ils mettent plus facilement de l'avant ce motif que ce n’était le cas auparavant.

Les obligations familiales ne ressortent donc pas comme une raison dominante pour le travail à domicile. Il convient de noter, par ailleurs, qu’outre les exigences du travail qui recueillent les deux tiers des réponses, les « autres » raisons sont évoquées par 30 \% des employés qui ont ont recours au travail à domicile. D’autres recherches sur le télétravail indiquent que l'économie de temps et d'argent est le motif dominant (Cefrio, 2001; Taskin et Vendramin, 2004), ce qui semble être également le cas ici pour un bon pourcentage de canadiennes et de canadiens.

Tableau 6

Les mesures d'aménagement du temps de travail, selon le sexe, 1999-2002

\begin{tabular}{|l|c|c|c|c|}
\hline & $\begin{array}{c}\text { Femmes } \\
\mathbf{1 9 9 9}\end{array}$ & $\begin{array}{c}\text { Hommes } \\
\mathbf{1 9 9 9}\end{array}$ & $\begin{array}{c}\text { Femmes } \\
\mathbf{2 0 0 2}\end{array}$ & $\begin{array}{c}\text { Hommes } \\
\mathbf{2 0 0 2}\end{array}$ \\
\hline Travail à la maison & 24,66 & 28,97 & 24,49 & 26,70 \\
\hline $\begin{array}{l}\text { Raisons travail maison : } \\
\text { Exigences du travail }\end{array}$ & 66,80 & 64,65 & 65,31 & 62,29 \\
\hline Obligations familiales* & 6,08 & 2,28 & 4,71 & 6,75 \\
\hline
\end{tabular}




\begin{tabular}{|l|c|c|c|c|}
\hline Autres** & 27,12 & 33,07 & 29,97 & 30,95 \\
\hline \hline Semaine comprimée & 6,08 & 12,88 & 5,25 & 7,93 \\
\hline Semaine de travail réduite & 15,98 & 10,35 & 10,33 & 4,90 \\
\hline \hline Horaire variable & 36,03 & 43,51 & 37,94 & 37,62 \\
\hline
\end{tabular}

*Obligations familiales regroupait les soins des enfants ou d'autres membres de la famille, ainsi que d'autres obligations personnelles ou familiales.

**Les autres raisons regroupent le fait de rechercher de meilleures conditions de travail, de gagner du temps et d'économiser de l'argent.

En ce qui concerne les aménagements de la semaine de travail, on note que moins de gens se prévalent de la semaine comprimée en 2002 que ce n'était le cas en 1999, le recul étant particulièrement marqué chez les hommes, passant de 12,88 \% à 7,93, alors que les femmes passaient de 6,08 à 5,25 \%. Rappelons les données du tableau 1 qui font ressortir le fait que, comparativement aux hommes, la semaine de travail moyenne des femmes est inférieure de 6 heures (leur semaine de travail étant de 33,88 heures contre 39,51 pour les hommes), de sorte qu'elles ont déjà en moyenne une journée de moins sur la semaine. On notera plus loin (tableau 7) que la présence d'enfants influe sur le recours à la semaine comprimée; celle-ci est effectivement plus fréquente chez les personnes avec enfant que chez les personnes sans enfant, les personnes ayant un ou deux enfant étant les plus fortes utilisatrices.

La semaine de travail réduite en vertu d'une entente spéciale avec l'employeur a aussi reculé chez les hommes comme chez les femmes, de sorte que cette mesure semble actuellement être moins disponible aux salariés ou moins recherchée par eux. Nous verrons toutefois plus loin que la semaine réduite trouve davantage preneur au fur et à mesure que le nombre d'enfants augmente.

Pour ce qui est de l'horaire variable ${ }^{5}$, il semble que les hommes en profitent un peu moins en 2002 comparativement à 1999, alors que les femmes en profitent un peu plus. Ce sont tout de même près de 40 \% des individus qui affirment avoir ce type d'horaire.

\footnotetext{
${ }^{5}$ ( l'horaire variable est défini ici comme la possibilité de faire varier ses heures d'arrivée et de départ, pourvu que l'on réalise un nombre donné d'heures de travail hebdomadaire, avec une plage fixe au centre de la journée)
} 
Le tableau 7 indique que le travail à domicile est plus fréquent chez les personnes ayant un ou deux enfants, et ces deux groupes donnent plus souvent la conciliation comme motif, le pourcentage de personnes ayant évoqué ce motif s'étant même accru de 1999 à 2002. Les exigences du travail demeurent au premier rang quel que soit le nombre d'enfants, mais la conciliation emploi-famille est le motif avancé par près de $15 \%$ des personnes ayant un enfant, et par $8,5 \%$ des personnes ayant deux enfants. Les pourcentages n'étaient que de près de $6 \%$ en 1999.

En ce qui concerne les aménagements de la semaine de travail, nous avons noté plus haut que moins de gens se prévalent de la semaine comprimée en 2002 que ce n'était le cas en 1999, mais les pourcentages restent toujours un peu plus élevés dans les groupes ayant un ou deux enfants.

Même si le recours à la semaine de travail réduite en vertu d'une entente spéciale avec l'employeur a diminué entre 1999 et 2002, on remarque, néanmoins, qu'il augmente clairement en fonction du nombre d'enfants, passant de 5,29 \% à 7,9 \% puis à 8,23 \% avec le passage de 1 à 2, puis 3 enfants.

Pour ce qui est de l'horaire variable (soit la possibilité de faire varier ses heures d'arrivée et de départ, il touche entre $35 \%$ et $40 \%$ des gens et augmente légèrement selon que le nombre d'enfants passe de 1 à 2 puis à 3 .

Tableau 7a

Les mesures d'aménagement du temps de travail, selon le nombre d'enfants, 1999

\begin{tabular}{|l|c|c|c|c|}
\hline \multicolumn{1}{|c|}{1999} & Aucun & 1 enfant & 2 enfants & 3 et plus \\
\hline \hline Travail à la maison & 23,35 & 26,76 & 33,56 & 29,77 \\
\hline \hline \begin{tabular}{l|c|c|c|}
\hline Raisons travail maison : \\
Exigences du travail
\end{tabular} & 63,76 & 69,50 & 67,22 & 64,20 \\
\hline \hline Obligations familiales* & 2,43 & 5,79 & 5,79 & 4,47 \\
\hline \hline Autres** & 33,81 & 24,71 & 27,00 & 31,33 \\
\hline Semaine comprimée & 7,78 & 12,49 & 8,83 & 8,56 \\
\hline
\end{tabular}




\begin{tabular}{|l|c|c|c|c|}
\hline Semaine de travail réduite & 13,04 & 12,71 & 15,52 & 16,28 \\
\hline Horaire variable & 40,39 & 37,50 & 39,06 & 40,20 \\
\hline
\end{tabular}

*Obligations familiales regroupait les soins des enfants ou d'autres membres de la famille, ainsi que d'autres obligations personnelles ou familiales.

**Les autres raisons regroupent le fait de rechercher de meilleures conditions de travail, de gagner du temps et d'économiser de l'argent.

\section{Tableau $7 \mathbf{b}$}

Les mesures d'aménagement du temps de travail, selon le nombre d'enfants 2002

\begin{tabular}{|c|c|c|c|c|}
\hline 2002 & Aucun & 1 enfant & 2 enfants & 3 et plus \\
\hline Travail à la maison & 21,72 & 26,20 & 30,61 & 33,64 \\
\hline $\begin{array}{l}\text { Raisons travail maison : } \\
\text { Exigences du travail }\end{array}$ & 63,34 & 53,34 & 67,50 & 71,62 \\
\hline Obligations familiales* & 0,71 & 14,93 & 8,48 & 4,70 \\
\hline Autres** & 35,94 & 31,74 & 24,01 & 23,67 \\
\hline Semaine comprimée & 6,21 & 6,31 & 7,94 & 5,71 \\
\hline Semaine de travail réduite & 8,18 & 5,29 & 7,90 & 8,23 \\
\hline Horaire variable & 38,23 & 35,81 & 37,45 & 39,82 \\
\hline
\end{tabular}

*Obligations familiales regroupait les soins des enfants ou d'autres membres de la famille, ainsi que d'autres obligations personnelles ou familiales.

**Les autres raisons regroupent le fait de rechercher de meilleures conditions de travail, de gagner du temps et d'économiser de l'argent.

\subsubsection{Autres mesures favorables à la conciliation}

Alors que l'aide à la garde et les services de garde chez l'employeur sont le premier souhait des parents canadiens ${ }^{6}$ ayant des enfants de moins de 3 ans (Lero et al., 1993), on constate qu’à peine

\footnotetext{
${ }^{6}$ Bien que l’on n’ait pas de données comparables et représentatives concernant uniquement le Québec, les enquêtes menées au Québec indiquent que les parents de jeunes enfants ne recherchent pas nécessairement un service de garde en entreprise, puisque le système des Centres de la petite enfance est bien développé au Québec et que les garderies
} 
plus du quart des travailleurs canadiens déclarent que leurs employeurs offrent un service à cet égard en 2002. De plus, il faut noter que la question de l'EMTE à cet égard est malheureusement très englobante puisqu'elle demande si l'employeur offre «de l'aide pour le gardiennage d'enfants soit par un centre sur place, ou par le biais de fournisseurs ou par des arrangements informels ». Le dernier élément est, on en conviendra, fort large, mais on voit mal quel type d'arrangement informel pourrait convenir aux parents sur une base régulière, car il faut bien faire garder ses enfants tous les jours lorsqu'on travaille tous les jours... De ce fait, on peut penser que ces pourcentages renvoient à un centre sur place ou un fournisseur externe essentiellement et que les arrangements informels en matière de garde doivent être assez peu fréquents.

Il est intéressant de noter, à partir du tableau 8, qu'il y a progression du pourcentage d'entreprises offrant ce type d'aide puisqu'en 1999, ce n'était qu'environ une personne sur cinq qui déclarait que son employeur offrait ce genre de service. Il est aussi intéressant de noter qu'entre 1999 et 2002, cette progression était manifeste autant pour les hommes que pour les femmes.

Notons par ailleurs, que si en 1999, les hommes, comparativement aux femmes, faisaient davantage appel au service d'aide pour la garde d'enfants, la situation s'est inversée en 2002 puisque l'on constate que 8,59 \% de femmes font appel à ce genre de service contre 6,21 \% des hommes.

Tableau 8

Les autres mesures de conciliation, selon le sexe, 1999-2002

\begin{tabular}{|l|c|c|c|c|}
\hline & $\begin{array}{c}\text { Femmes } \\
\mathbf{1 9 9 9}\end{array}$ & $\begin{array}{c}\text { Hommes } \\
\text { 1999 }\end{array}$ & $\begin{array}{c}\text { Femmes } \\
\mathbf{2 0 0 2}\end{array}$ & $\begin{array}{c}\text { Hommes } \\
\mathbf{2 0 0 2}\end{array}$ \\
\hline \begin{tabular}{l|c|c|c|}
\hline Existence d'aide pour la garde \\
d'enfants
\end{tabular} & 20,75 & 18,03 & 29,51 & 26,46 \\
\hline $\begin{array}{l}\text { Recours à l'aide pour la garde } \\
\text { d'enfants }\end{array}$ & 5,30 & 6,11 & 8,59 & 6,21 \\
\hline $\begin{array}{l}\text { Existence de services de soins } \\
\text { aux aînés }\end{array}$ & 12,36 & 10,35 & 12,89 & 12,80 \\
\hline
\end{tabular}

de voisinage sont assez nombreuses, tout en étant soutenues par l'État (services de garde à contribution réduite, soit à 7 \$ par jour en 2005). Aussi, la présence ou l'absence de service de garde ne ressort pas comme variable déterminante des difficultés de conciliation ou comme facteur facilitateur dans les recherches menées sur le sujet au Québec. Source : Tremblay, 2004. 


\begin{tabular}{|l|c|c|c|c|}
\hline $\begin{array}{l}\text { Recours aux services de soins } \\
\text { aux aînés }\end{array}$ & 5,98 & 11,75 & 6,54 & 1,87 \\
\hline
\end{tabular}

Il y a lieu d'ajouter que l'on se préoccupe de plus en plus de ce qu'il est convenu d'appeler la génération Sandwich, en référence à un article récent de Statistique Canada, indiquant que de plus en plus de parents canadiens étaient simultanément concernés par les difficultés de garde de jeunes enfants, et par les soins à leurs parents âgés. À cet égard, l’EMTE a interrogé les travailleurs sur l'offre par leur employeur de « services d'aide pour les soins aux aînés » (c’est la formulation exacte de la question, qui n’est pas très précise, mais donne tout de même une première indication sur cette question peu étudiée).

On observe ainsi que l'offre de tels services a un peu progressé dans les organisations, se maintenant autour de 12 \%; le recours a apparemment augmenté chez les femmes, mais les données indiquent une forte baisse chez les hommes, ce qui étonne quelque peu.

Si nous analysons la question selon qu'il y a présence d'enfants ou non, et selon le nombre d'enfants, on constate qu'entre 1999 et 2002, il y a forte progression de l'offre de services concernant l'aide à la garde des enfants dans les groupes n'ayant pas d'enfants ou ayant un enfant. Ceux-ci sont vraisemblablement les plus jeunes et pourraient créer une famille dans les années à venir; ils pourraient d'ailleurs avoir choisi un employeur en partie pour cette raison, comme l'indiquent certaines recherches. Cette question mériterait d’être analysée plus en détail, mais les données de l’EMTE permettent au moins un premier éclairage.

L’offre de services de soins aux aînés a aussi légèrement progressé et en 2002, elle est plus forte dans les groupes ayant un ou trois enfants. Les données sur le recours à cette mesure ne sont pas disponibles ${ }^{7}$.

\footnotetext{
${ }^{7}$ Le nombre de répondants de l'échantillon non pondéré de 23000 personnes est inférieur à cinq et de ce fait, les données ne peuvent être divulguée par soucis de conserver leur confidentialité.
} 
Tableau 9a

Les autres mesures de conciliation, selon le nombre d'enfants, 1999

\begin{tabular}{|l|c|c|c|c|}
\hline \multicolumn{1}{|c|}{1999} & Aucun & 1 enfant & 2 enfants & 3 et plus \\
\hline $\begin{array}{l}\text { Existence d'aide pour la } \\
\text { garde d'enfants }\end{array}$ & 17,82 & 16,23 & 22,90 & 22,91 \\
\hline $\begin{array}{l}\text { Existence de services } \\
\text { de soins aux aînés }\end{array}$ & 12,80 & 8,01 & 11,20 & 9,64 \\
\hline
\end{tabular}

Tableau 9b

Les autres mesures de conciliation, selon le nombre d'enfants, 2002

\begin{tabular}{|c|c|c|c|c|}
\hline 2002 & Aucun & 1 enfant & 2 enfants & 3 et plus \\
\hline $\begin{array}{l}\text { Existence d'aide pour la } \\
\text { garde d'enfants }\end{array}$ & 33,12 & 26,62 & 18,50 & 28,77 \\
\hline $\begin{array}{l}\text { Existence de services } \\
\text { de soins aux aînés }\end{array}$ & 15,26 & 12,76 & 9,35 & 9,93 \\
\hline
\end{tabular}

\section{Discussion}

Les données présentées dans cet article indiquent dans l'ensemble que les progrès observés en ce qui concerne le débat social sur la conciliation travail-famille ne se sont pas nécessairement traduits par une amélioration notable des conditions favorables à la conciliation dans les milieux de travail. Qui plus est, à certains égards, il y a même eu des reculs, rendant la conciliation d'exigences conflictuelles encore plus difficile.

En ce qui a trait aux heures de travail, les mouvements des quatre années étudiées ne connaissent pas de sens unique. On note, toutefois, que les femmes travaillent moins d'heures que les hommes, qu'elles sont plus nombreuses que les hommes à souhaiter réduire leurs heures de travail et que c'est plus souvent pour des raisons familiales. Au cours de la même période, on note que les heures supplémentaires non rémunérées ou rémunérées se sont réduites. Par contre, 
le nombre de jours de travail par semaine s'est légèrement accru, se rapprochant fortement de 5 jours en moyenne pour les hommes et de 4,6 jours pour les femmes en 2002.

Nous pouvons aussi ajouter qu'un pourcentage important de la main-d'œuvre canadienne vit des horaires de travail variables et des horaires rotatifs, ce qui a été identifié comme source de difficultés de conciliation dans d'autres recherches. Par ailleurs, une bonne partie des travailleuses et travailleurs canadiens déclare travailler un certain nombre d'heures à domicile, ce qui peut favoriser la conciliation, mais peut aussi être source d'empiètement sur la vie privée. Les données de l'EMTE sont éloquentes à cet égard dans la mesure où elles montrent clairement que les gens ne travaillent pas à la maison pour pouvoir mieux concilier leurs obligations, mais plutôt parce que le travail l'exige. En somme, on assiste ici à un débordement du travail sur la vie personnelle au lieu d'une pratique qui offre à l'individu plus de flexibilité dans l'organisation de son temps de vie.

Par ailleurs, notons que si l'aide à la garde et les services de garde chez l'employeur sont le premier souhait des parents canadiens ayant des enfants de moins de 3 ans, on constate qu'à peine plus du quart des travailleurs canadiens déclarent que leurs employeurs offrent un service à cet égard en 2002. Aussi, on a pu observer que l'offre de services de soins aux aînés ne touche qu'un dixième des travailleuses et des travailleurs canadiens.

En somme, aujourd'hui, on a l'impression que dans les organisations, la gestion relève encore d'un modèle organisationnel présumant que les responsabilités familiales constituent un domaine qui ne concerne pas l'employeur. Il semble que l'articulation emploi-famille, même si l'on en parle sur la scène publique, reste du domaine de la vie privée des employés, ou encore, dans certains milieux, une «affaire de femmes», plutôt qu'une « affaire de société » Pourtant, le problème de l'articulation emploi-famille ne peut se poser qu'avec plus d'acuité dans l'avenir, puisque les femmes sont de plus en plus actives à plein temps et que la progression est particulièrement marquée chez les femmes avec enfants. Les aménagements au sein de la famille seront donc de plus en plus complexes à réaliser. Ainsi, pour assurer une intégration équitable des femmes sur le marché du travail et une participation équitable des hommes aux responsabilités parentales, il importe d'identifier les facteurs et mesures pouvant faciliter la conciliation emploi-famille. À cette fin, il serait utile de 
procéder, ultérieurement, à des analyses économétriques plus poussées pour identifier les facteurs explicatifs de l'incidence des mesures de conciliation travail-famille. 


\section{Références bibliographiques}

Baines, S. et U. Gelder (2003). What is family friendly about the workplace in the home ? The Case of self-employed parents and their children. Technology, Work and Employment, 18(3), 223-234.

Carlson, Dawn, S., K. Michele Kaemar, et Larry J. Williams. 2000. « Construction and Initial Validation of a Multidimensional Measure of Work-Family Conflict », Journal of Vocational Behavior, 56 : 249-276.

Descarries, Francine, et Christine Corbeil (sous la direction de). 1995. Ré/conciliation familletravail : les enjeux de la recherche. Montréal: Université du Québec.

Descarries, Francine, et Christine Corbeil. 1996. La conciliation travail-famille. Dans Science, conscience et action : 25 ans de recherche féministe au Québec, sous la direction de H. Dagenais. Montréal: Les Éditions du Remue-Ménage.

Descarries, Francine, Christine Corbeil, Carmen Gill, et Céline Séguin. 1995a. Travail et vie familiale : Une difficile articulation pour les mères en emploi. Montréal: Université du Québec à Montréal: Institut de recherches et d'études féministes.

Descarries, Francine, Christine Corbeil, Carmen Gill, et Céline Séguin. 1995b. Famille et travail : Double statut... double enjeu pour les mères en emploi. Montréal: Université du Québec à Montréal: Institut de recherches et d'études féministes.

Descarries, Francine, Christine Corbeil, Carmen Gill, et Céline Séguin. 1995c. Famille et travail : Un double statut... un double enjeu pour les mères en emploi. Cahiers réseau de recherches féministes (3):161-182.

Duxbury, L.E., C.A. Higgins et C. Lee (1994). «Work-Family Conflict. A Comparison by Gender, Family Type and Perceived Control», Journal of Family Issues, 15(3), p. 449-466.

Duxbury, Linda Elizabeth, Christopher Alan Higgins, et C. Lee. (1993). « The Impact of Job Type and Family Type on Work-Family Conflict and Perceived Stress : A Comparative Analysis », Ressources humaines, ASAC'93, 14(9) : 21-29. 
Fagnani, Jeanne (2000). Un travail et des enfants. Petits arbitrages et grands dilemmes, Paris: Bayard. 2000.

Fagnani, J. et M.-T. Letablier (2001), Famille et travail : contraintes et arbitrages, Problémes politiques et sociaux, $\mathrm{n}^{\circ}$ 858, juin 2001.

Families and Work Institute (1997). 1997 National Study of the Changing Workforce, New York : Families and Work Institute. http://www.familiesandwork.org

Families and Work Institute (1998).1998 Business Work-Life Study. Families and Work Institute http://www.familiesandwork.org

Frederick, Judith A., et Janet E. Fast. 2001. « Aimer son travail : une stratégie efficace pour équilibrer la vie professionnelle et la vie privée? », Tendances sociales canadiennes, été, $9-13$.

Galinsky, Ellen, Stacy S. Kim, et James T. Bond. 2001. Feeling Overworked: When Work Becomes too Much - Executive Summary, New York: Families and Work Institute. http://www.familiesandwork.org

Guérin, Gilles, Sylvie St-Onge, Laure Chevalier, Kathy Denault, et Martine Deschamps. 1997. Le conflit emploi-famille: ses causes et ses conséquences: Résultats d'enquête, Montréal : Université de Montréal, École de relations industrielles, 23 pages.

Guérin, Gilles, Sylvie St-Onge, Renée Trottier, Victor Haines et Manon Simard (1994). «Les pratiques organisationnelles d'aide à la gestion de l'équilibre travail-famille: la situation au Québec», Gestion, 19(2), p. 74-82.

Lero, Donna S., Lois M. Brockman, Alan R. Pence, Hillel Goelman et Karen L. Johnson (1993). Étude nationale canadienne sur la garde des enfants: Avantages et flexibilité en milieu de travail: tour d'horizon des expériences vécues par les parents, Ottawa, Statistique Canada, cat. 89-530F. 
Nelson, Debra L., et James C. Quick. 1985. «Professional Women : Are Distress and Disease Inevitable? », Academy of Management Review, 19(2) : 206-218.

Paquet, Renaud et Elmustapha Najem. 2005. «L’évolution récente des pratiques de conciliation travail-famille dans les entreprises canadiennes ", Actes du Congrès relations de travail et organisations (en attente de publication).

Najem, Elmustapha et Renaud Paquet. (2005). « Étude comparative des pratiques de gestion des ressources humaines dans les milieux syndiqués et non-syndiqués ", Actes du Congrès de l'Association canadienne des relations industrielles (en attente de publication).

Politiques sociales. Numéro spécial sur la conciliation emploi-famille. Bruxelles et Madrid. Vol. 63, no 3-4. Automne 2003.

Pronovost, G. (2005). La conciliation famille-travail et l'aménagement du temps dans Tremblay, D.-G. (2005, dir). De la conciliation emploi-famille à une politique des temps sociaux. Québec : Presses de l’université du Québec. Pp.

Statistique Canada. 2004. Guide pour l'analyse de l'enquête sur le milieu de travail et les employés. www.statcan.ca:8096/bsolc/francais/bsolc?catno=71-221-G\&CHROPG=1

Taskin, L. et P. Vendramin (2004). Le télétravail; une vague silencieuse. Louvain : Presses universitaires de Louvain.114 p.

Tremblay, D.-G. (2004). Conciliation emploi-famille et temps sociaux. Québec-Toulouse: Presses de l’Université du Québec et Octares. 340 p.

Tremblay, D.-G. (2004a). Economie du travail. Les réalités et les approches théoriques.(Édition revue) Montréal: Editions Saint-Martin/Télé-Université. 482 p.

Tremblay, D.-G. (2004b). Articulation emploi-famille et temps de travail: Les usages différenciés du temps chez les pères et les mères. Nouvelles pratiques sociales. Vol. 16 no 1. pp. 7693.

Tremblay, D.-G. (2003). Articulation emploi-famille : Comment les pères voient-ils les choses ? Politiques sociales. Bruxelles et Madrid. Vol. 63, no 3-4. Automne 2003. Pp.70-86. 
Tremblay, D.-G. (2002). Balancing Work and Family with Telework? Organizational Issues and Challenges for Women and Managers. In Women in Management. Manchester: MCB Press. Volume 17 issue 3/4. pp157-170.

Tremblay, D.-G. (2002). dans Tremblay, D.-G. et L.-F. Dagenais (2002). Articulation emploifamille et temps de travail; comment concilier famille et travail dans les secteurs à horaires variables ? Segmentations, ruptures et mutations du marché du travail. Québec : Presses de l’université du Québec. Pp.

Tremblay, D.-G. et M. De Sève (2002). Articulation emploi-famille et temps de travail ; résultats de l'analyse qualitative dans les secteurs de la santé et de l'éducation. Rapport de recherche.

Tremblay, D.-G. et Amherdt, C.H. (2000). La vie en double. Les obstacles organisationnels et socioculturels à la conciliation des responsabilités parentales et professionnelles chez les pères et les mères. Rapport de recherche. 99 p. Texte 2.3 accessible sur le site du cours RIN 2013 sur le site www.teluq.uquebec.ca . 